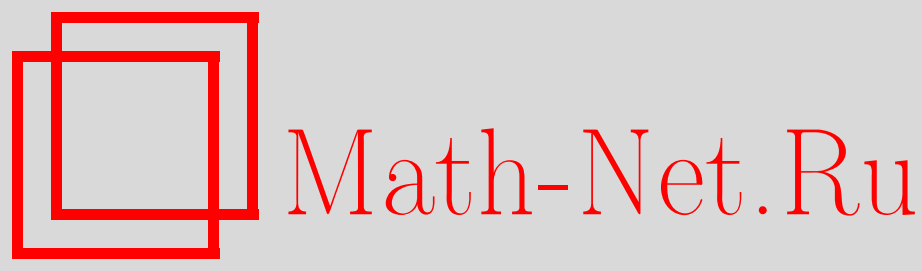

П. Зинн-Жюстен, П. Ди Франческо, Квантовое уравнение Книжника-Замолодчикова, полностью симметричные самодополнительные разбиения плоскости и матрицы чередующихся знаков, ТМФ, 2008, том 154, номер 3, 387-408

DOI: https://doi.org/10.4213/tmf6178

Использование Общероссийского математического портала Math-Net.Ru подразумевает, что вы прочитали и согласны с пользовательским соглашением http://www . mathnet.ru/rus/agreement

Параметры загрузки:

IP : 54.197 .130 .99

26 апреля 2023 г., 16:59:32

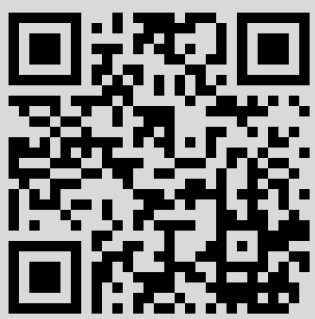




\title{
КВАНТОВОЕ УРАВНЕНИЕ КНИЖНИКА- ЗАМОЛОДЧИКОВА, ПОЛНОСТЬЮ СИММЕТРИЧНЫЕ САМОДОПОЛНИТЕЛЬНЫЕ РАЗБИЕНИЯ ПЛОСКОСТИ И МАТРИЦЫ ЧЕРЕДУЮЩИХСЯ ЗНАКОВ
}

\begin{abstract}
Представлены интегральные формулы с множественными вычетами для частичных сумм в базисе типов спаривания полиномиальных решений квантового уравнения Книжника-Замолодчикова уровня 1 для $U_{q}\left(\widehat{\mathfrak{s l}_{2}}\right)$ при произвольных значениях квантового параметра $q$. Эти уравнения позволяют переформулировать и обобщить недавнюю гипотезу Ди Франческо о связи этих сумм с производящими многочленами для взвешенных полностью симметричных самодополнительных разбиений плоскости. Соответствующие гипотезы сведены к одному интегральному тождеству, которое еще предстоит доказать.
\end{abstract}

Ключевые слова: петлевые модели, комбинаторика, квантовая интегрируемость.

\section{1. ВВЕДЕНИЕ}

В последние несколько лет мы являлись свидетелями обнаружения множества взаимоотношений, всевозрастающей сложности, между различными комбинаторными объектами и некоторыми интегрируемыми моделями: с одной стороны, это матрицы чередующихся знаков (МЧЗ), плотные упаковки петель и разбиения плоскости, с другой - $X X Z$-цепочка и связанные с ней спиновые цепочки, а также решеточные петлевые модели. Целью данной работы является разработка некоторых методов доказательства комбинаторных гипотез, которые были сформулированы ранее, в частности для доказательства гипотезы из работы [1]. Мы полагаем, что развиваемые здесь методы могут быть с равным успехом приложены к ряду других случаев, в частности к случаю других граничных условий в петлевой модели (например, для открытых границ, см. гипотезы в работе [2]).

* Laboratoire de Physique Théorique et Modèles Statistiques, Univ Paris-Sud, Orsay, France. E-mail: paul.zinn-justin@u-psud.fr

${ }^{\dagger}$ Service de Physique Théorique de Saclay, Gif sur Yvette Cedex, France 


\section{1. Интегрируемая петлевая модель и гипотеза Разумова-Строганова.}

Петлевая модель Темперли-Либа определена на полубесконечном цилиндре, представляющем собой квадратную решетку с четным периметром $2 n$ и с центрами ребер, занумерованными как $1,2, \ldots, 2 n$ против часовой стрелки. Конфигурации модели получаются выбором любой из двух возможных конфигураций каждой грани решетки. Можно видеть, что конфигурации модели образуют или замкнутые петли, или разомкнутые кривые, попарно соединяющие граничные точки, без всяких пересечений кривых. В действительности каждая конфигурация порождает планарное спаривание граничных точек, характеризуемое типом спаривания, а именно диаграммой, в которой $2 n$ занумерованных и равно отстоящих друг от друга точек на окружности спарены непересекающимися прямыми отрезками. Множество типов спаривания для $2 n$ точек обозначается как $\mathcal{L}_{2 n}$, а его мощность есть $c_{n}=(2 n) ! /(n !(n+1) !)$. Мы также рассматриваем $\pi \in \mathcal{L}_{2 n}$ как инволюцию $\mathcal{S}_{2 n}$ без фиксированных точек.

Мы будем рассматривать неоднородную модель, в которой с конфигурациями граней связываются вероятности, зависящие от номера столбца $i$, в которую они вставляются. Используем следующую параметризацию вероятностей в терминах переменных $z_{i}$ :

$$
T_{n}\left(t ; z_{1}, \ldots, z_{2 n}\right)=\prod_{i=1}^{2 n}\left(\frac{q z_{i}-q^{-1} t}{q t-q^{-1} z_{i}} \square+\frac{z_{i}-t}{q t-q^{-1} z_{i}} \square \Omega\right)
$$

где $T$ - трансфер-матрица, которая добавляет одну строку к полубесконечному цилиндру, а $q=e^{2 i \pi / 3}$. Переменная $t$ является дополнительной, ее можно включить в $z_{i}$; однако ее полезно ввести, поскольку при фиксированных $z_{i}$ вследствие интегрируемости справедливы коммутационные соотношения $\left[T_{n}(t), T_{n}\left(t^{\prime}\right)\right]=0$.

Теперь можно задать вопрос: какова вероятность $P_{\pi}\left(z_{1}, \ldots, z_{2 n}\right)$ того, что граничные точки случайной конфигурации модели будут соединены парами в соответствии с данным типом спаривания $\pi \in \mathcal{L}_{2 n}$ ? Составив вектор $P_{n}\left(z_{1}, \ldots, z_{2 n}\right)$ с компонентами $P_{\pi}\left(z_{1}, \ldots, z_{2 n}\right)$ в векторном пространстве с каноническим базисом, проиндексированным типами спаривания $\pi$, мы немедленно видим, что он удовлетворяет условию на собственные векторы

$$
T_{n}\left(t ; z_{1}, \ldots, z_{2 n}\right) P_{n}\left(z_{1}, \ldots, z_{2 n}\right)=P_{n}\left(z_{1}, \ldots, z_{2 n}\right) .
$$

Уравнение (2) не фиксирует нормировку $P_{n}$, которая определяется тем, что полная вероятность равна единице. Однако удобнее рассматривать другую нормировку решения уравнения (2), которое мы обозначим как $\Psi_{n}$, с компонентами $\Psi_{\pi}$, являющимися взаимно простыми многочленами по переменным $z_{i}$. При этом сохраняется произвол в умножении на численную постоянную, который мы фиксируем позже.

Основные гипотезы касаются однородного предела $z_{1}=\cdots=z_{2 n}=1$. В работе [3] было замечено следующее:

1) все величины $\Psi_{\pi} / \Psi_{\pi_{0}}$, где $\Psi_{\pi_{0}}$ - наименьшая компонента, являются положительными целыми числами;

2) $\Psi_{\max } / \Psi_{\pi_{0}}=A_{n-1}$, где $\Psi_{\max }-$ наибольшая компонента; 
3) $\sum_{\pi \in \mathcal{L}_{2 n}} \Psi_{\pi} / \Psi_{\pi_{0}}=A_{n}$,

где $A_{n}$ - число МЧЗ размера $n$ (их историю и ссылки см. в книге Бресу [4]):

$$
A_{n}=\frac{1 ! 4 ! 7 ! \ldots(3 n-2) !}{n !(n+1) !(n+2) ! \ldots(2 n-1) !} .
$$

Число $A_{n}$ равно также числу полностью симметричных самодополнительных разбиений плоскости (ПССДРП), которые можно рассматривать как замощения правильного шестиугольника с размером ребра $2 n$ на треугольной решетке элементарными ромбами, при этом рассматриваются конфигурации, обладающие всеми возможными симметриями шестиугольника. До недавнего времени казалось, что этот факт не имеет прямого отношения к данной модели, особенно ввиду гипотезы Разумова-Строганова [5], которая утверждает, что $\Psi_{\pi} / \Psi_{\pi_{0}}$ есть число полностью упакованных петлевых (ПУП) конфигураций с типом спаривания $\pi$. Последние являются конфигурациями петель, проведенных по ребрам квадратной сетки размера $n \times n$ так, что в каждой вершине ровно два ребра заняты ребрами петель, и с граничным условием, что каждое второе ребро на границе занято. Нумерация этих ребер посредством чисел $1,2, \ldots, 2 n$ позволяет следить за их спариваниями посредством кривых и ассоциировать с каждым типом спаривания $\pi$ множество ПУП-конфигураций. В силу их определения, ПУП-конфигурации находятся в очевидном взаимно однозначном соответствии с конфигурациями шестивершинной модели с граничными условиями типа доменной стенки, а также с МЧЗ. Поэтому гипотеза Разумова-Строганова дает интерпретацию гипотезам 1 и 3 (и в принципе также и гипотезе 2) в терминах перечислений. Заметим, что, напротив, не известно взаимно однозначного соответствия между МЧЗ и ПССДРП.

Гипотеза 3 была доказана в работе [6] путем ее обобщения на неоднородную модель в виде $\sum_{\pi} \Psi_{\pi}=Z_{n}\left(z_{1}, \ldots, z_{2 n}\right)$, где $Z_{n}$ - статистическая сумма ИзергинаКорепина шестивершинной модели с граничными условиями типа доменной стенки [7], которая возникала в работе [8] в контексте перечисления МЧЗ.

\section{2. Гипотеза ПССДРП и минимальные полиномиальные решения} квантового уравнения Книжника-Замолодчикова. В работе [6] было отмечено, что уравнение (2) можно решить, записывая вместо него следствия, которые вытекают для $\Psi_{n}$ из сплетающих соотношений для трансфер-матрицы (1) неоднородной петлевой модели. В конце концов их можно записать в виде квантового уравнения Книжника-Замолодчикова $(q \mathrm{~K} 3)$ уровня 1 для $U_{q}\left(\widehat{\mathfrak{s l}_{2}}\right)$ [9] на вектор основного состояния $\Psi_{n}$ при $q=-e^{i \pi / 3}$. При записи в компонентах это уравнение позволяет с помощью нижнетреугольного преобразования выразить все полиномиальные компоненты $\Psi_{\pi}$ через $\Psi_{\pi_{0}}$. При этом $\Psi_{\pi_{0}}$ является многочленом минимальной степени, подчиненным всем свойствам делимости, наследуемым из сплетающих соотношений, и имеет приятный факторизованный вид. Уравнение $q \mathrm{~K} 3$, которому удовлетворяет $\Psi_{n}$, можно альтернативным образом интерпретировать как утверждение о том, что компоненты $\Psi_{\pi}$ образуют полиномиальное представление аффинной алгебры Темперли-Либа с весом каждой петли, равным $\tau=-q-q^{-1}=1$.

Имеется естественная $q$-деформация задачи, получаемая или путем поиска полиномиальных представлений аффинной алгебры Темперли-Либа с весом $\tau=-q-q^{-1}$ 
для каждой петли [10], или, что эквивалентно, путем поиска полиномиальных решений уравнений $q \mathrm{~K} 3$ уровня 1 для $U_{q}\left(\widehat{\mathfrak{s l}_{2}}\right)$ в случае, когда $q$ произвольно [11]. В работах [11], [12] было отмечено, что должным образом нормированный вектор $\Psi_{n}$ демонстрирует хорошие комбинаторные свойства в однородном пределе, но на этот раз как функция $\tau$. Конкретно говоря, было замечено, что каждое отношение $\Psi_{\pi} / \Psi_{\pi_{0}}$ при $z_{i}=1$ является многочленом по $\tau$ с неотрицательными целыми коэффициентами. Очень соблазнительно, конечно, было бы вывести существование некоторой $q$-деформированной гипотезы Разумова-Строганова, в которой эти величины можно было бы интерпретировать как взвешенные суммы ПУП-конфигураций с фиксированными типами спаривания.

В качестве первого шага в этом направлении, который был сделан в работе [1], была выдвинута гипотеза о сумме всех компонент в виде многочлена от $\tau$. Но неожиданным образом оказалось, что правильным комбинаторным объектом, который можно подозревать в том, что он лежит в основе этой суммы, являются не ПУП-конфигурации или МЧЗ, а скорее ПССДРП ${ }^{1)}$.

Чтобы понять эту гипотезу, необходимо сначала выразить ПССДРП как множество непересекающихся решеточных путей (НПРП), заметив для этого, что элементы замощения в фундаментальной области шестиугольника можно организовать во множество непересекающихся ломаных линий, распространяющихся в пределах данной области. Коль скоро это сделано, ПССДРП можно рассматривать как конфигурации решеточных путей, которые при представлении на квадратной решетке могут иметь два типа шагов, скажем вертикальные и диагональные.

Гипотеза ПССДРП утверждает:

4) $\sum_{\pi} \Psi_{\pi} / \Psi_{\pi_{0}}$ есть производящий многочлен для ПССДРП размера $2 n$ с весом $\tau$ для каждого вертикального шага в их формулировке в терминах НПРП.

Заметим, что гипотеза 4 сводится к гипотезе 3 при $\tau=1$. Основная цель данной работы - рассмотрение гипотезы ПССДРП.

Интересно отметить, что детальные перечисления ПССДРП ранее уже связывались с детальными перечислениями МЧЗ [14], но это всегда относилось к "граничным" взвешиваниям конфигураций (например, вес приписывался только последнему или первому шагу в ПССДРП, вместо того чтобы следить за положениями единиц в первой строке или первом столбце МЧЗ). Мы же здесь имеем "объемное" взвешивание, которое не выделяет граничные шаги. Вопрос о нахождении хорошей интерпретации для $\tau$-взвешивания в МЧЗ или ПУП-конфигурации остается открытым, и исследование этого вопроса может пролить свет на возможное взаимно однозначное соответствие между МЧЗ и ПССДРП.

1.3. Структура работы. В данной работе мы сначала рассматриваем взвешенное перечисление ПССДРП (раздел 2). Результаты получаются путем использования формулировки ПССДРП в терминах НПРП и знаменитой детерминантной формулы Линдстрома-Гесселя-Вьено (ЛГВ) [15] и принимают вид кратных контурных интегралов, которые берутся с помощью вычетов.

\footnotetext{
1) В действительности в некоторых более ранних работах [13] уже были обнаружены определенные связи между петлевой моделью в слегка деформированных цилиндрических геометриях и детальным перечислением ПССДРП, но при $\tau=1$.
} 


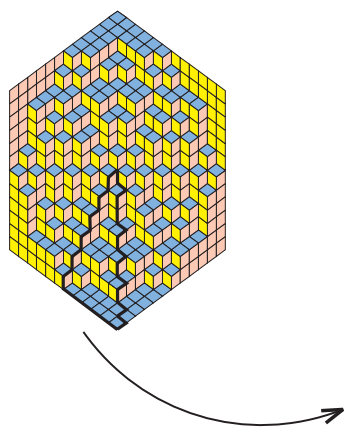

ПССДРП
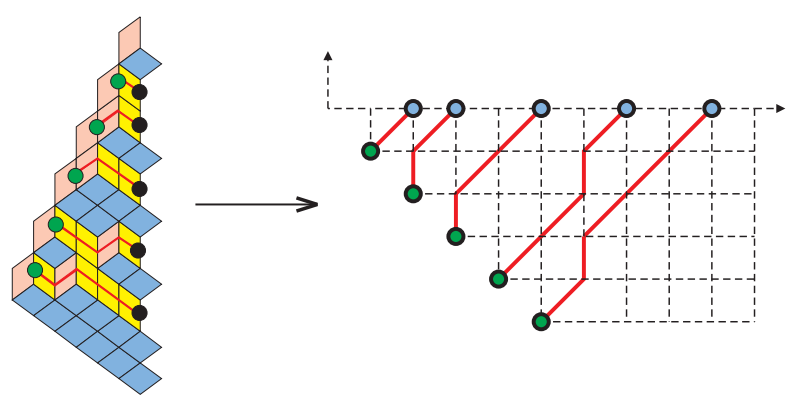

НПРП

Рис. 1. От ПССДРП к НПРП. После присвоения каждому вертикальному шагу в $i$-м (считая сверху) горизонтальном срезе веса $t_{i}$ НПРП-конфигурация приобретает вес $t_{2}^{2} t_{3} t_{4}$.

Затем в разделе 3 мы представляем пространство полиномиальных решений уравнения $q \mathrm{~K} 3$ в терминах кратных контурных интегралов с использованием нового набора векторов, индексируемых последовательностями целых чисел (отличным от так называемого спинового базиса, используемого на языке соответствующих спиновых цепочек). При переходе к базису петель (типов спаривания) это дает интегральные формулы для частичных сумм компонент вектора $\Psi$. В частности, мы получаем интегральные формулы для максимальной компоненты $\Psi_{\max }$, а также для суммы всех компонент $\sum_{\pi} \Psi_{\pi}$. Подробности смены базиса от этого нового множества векторов к типам спаривания даются в приложении А, а связь с компонентами в спиновом базисе рассмотрена в приложении Б.

В разделе 4 мы показываем, каким образом гипотеза ПССДРП (гипотеза 4), равно как и приведенные выше гипотезы 2 и 3, являются следствиями простого интегрального тождества с множественными вычетами, доказательство которого мы оставляем на будущее.

\section{2. ПОЛНОСТЬЮ СИММЕТРИЧНЫЕ САМОДОПОЛНИТЕЛЬНЫЕ РАЗБИЕНИЯ ПЛОСКОСТИ}

2.1. Определения. ПССДРП - это замощения ромбами правильного шестиугольника размера $2 n \times 2 n \times 2 n$ на треугольной решетке, обладающие вращательной и отражательной симметриями шестиугольника и реализующие свойство самодополнительности: если интерпретировать разбиение как перспективный вид горки элементарных кубов в положительном квадранте $\mathbb{Z}^{3}$ при рассмотрении в направлении $(1,1,1)$, то дополнение этой горки до куба размером $2 n$ является ее тождественной копией.

Это позволяет ограничить ПССДРП на фундаментальную область, составленную из (1/12)-й части исходного шестиугольника (см. рис. 1), и пересчитывать их, используя НПРП. Последние просто получить, рассматривая следующие друг 
за другом элементы двух из трех возможных типов, используемых при замощении фундаментальной области. Слегка деформируя геометрию, приходим к задаче пересчета $n-1$ НПРП на квадратной решетке, где каждый путь состоит только из вертикальных шагов вида $(0,1)$ или диагональных шагов вида $(1,1)$, причем $i$-й путь начинается в точке $(i,-i)$ и заканчивается на линии $y=0$ после $i$ шагов. В результате полное число таких путей равно $N_{10}(2 n)$, как это следует, например, из формулы ЛГВ [15], выражающей число НПРП с фиксированными концами в виде определителя матрицы, образованной числами путей из $i$-й начальной точки в $j$-ю конечную точку, скажем $\left(r_{j}, 0\right)$, с суммированием по положениям конечных точек. В работе [1] была предложена детализация этих чисел, при которой каждый вертикальный шаг считается с весом $\tau$; она описывается производящим многочленом $N_{10}(2 n \mid \tau)$ для ПССДРП, который дается следующей формулой ЛГВ:

$$
N_{10}(2 n \mid \tau)=\sum_{\substack{1 \leqslant r_{1}<r_{2}<\cdots<r_{n-1} \\
r_{i} \leqslant 2 i}} \operatorname{det}_{1 \leqslant i, j \leqslant n-1}\left(\left(\begin{array}{c}
i \\
r_{j}-i
\end{array}\right) \tau^{2 i-r_{j}}\right) .
$$

ПРимеР 1. При $n=3$ имеется семь конфигураций ПССДРП:

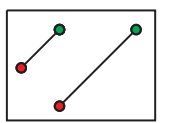

1

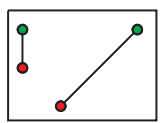

$\tau$

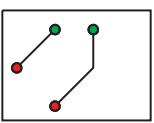

$\tau$

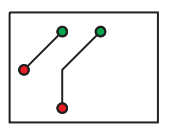

$\tau$

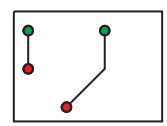

$\tau^{2}$

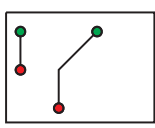

$\tau^{2}$

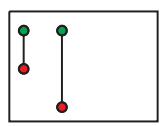

$\tau^{3}$

и в результате получается многочлен $N_{10}(6 \mid \tau)=1+3 \tau+2 \tau^{2}+\tau^{3}$.

2.2. Интегральные формулы. Выведем простую многоконтурную интегральную формулу для производящего многочлена $N_{10}\left(t_{1}, t_{2}, \ldots, t_{n-1}\right)$ для ПССДРП в их формулировке в терминах НПРП с весом $t_{i}$ на каждый вертикальный шаг в $i$-м срезе, ограниченном линиями $y=1-i$ и $y=-i$. Мы снова используем формулу ЛГВ со взвешиванием путей с весами $t_{i}$, которая имеет вид

$$
N_{10}\left(t_{1}, t_{2}, \ldots, t_{n-1}\right)=\sum_{\substack{1 \leqslant r_{1}<r_{2}<\cdots<r_{n-1} \\ r_{i} \leqslant 2 i}} \operatorname{det}_{1 \leqslant i, j \leqslant n-1} \mathcal{P}_{i, r_{j}},
$$

где $\mathcal{P}_{i, r}$ - взвешенная сумма по всем решеточным путям из $(i,-i)$ в $(r, 0)$. Такой путь имеет $r-i$ диагональных и $2 i-r$ вертикальных шагов, которые надо совершить в пределах первых $i$ срезов, и поэтому

$$
\mathcal{P}_{i, r}=\sum_{1 \leqslant i_{1}<i_{2}<\cdots<i_{2 i-r}} \prod_{\ell=1}^{2 i-r} t_{i_{\ell}}=\left.\prod_{k=1}^{i}\left(1+t_{k} u\right)\right|_{u^{2 i-r}}
$$

где нижний индекс указывает на коэффициент при соответствующей степени $u$ в многочлене. Вводя дополнительный тривиальный путь, начинающийся и заканчивающийся в начале координат, для которого $\mathcal{P}_{i, 0}=\delta_{i, 0}$, и подставляя (6) в (5), получаем следующее тождество для коэффициентов:

$$
N_{10}\left(t_{1}, t_{2}, \ldots, t_{n-1}\right)=\left.\prod_{1 \leqslant i<j \leqslant n} \frac{\left(u_{j}-u_{i}\right)\left(1+t_{i} u_{j}\right)}{1-u_{i} u_{j}} \prod_{i=1}^{n} \frac{1}{1-u_{i}}\right|_{\prod_{i=1}^{n} u_{i}^{2 i-2}},
$$




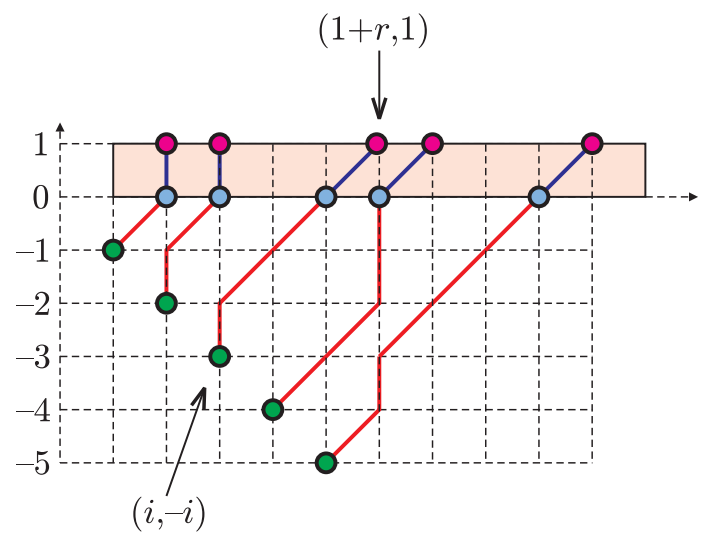

Рис. 2. НПРП для модифицированных ПССДРП. Мы добавили к исходным НПРП, изображенным на рис. 1, дополнительный верхний срез из шагов, так что теперь НПРП заканчиваются на линии $y=1$. Конечные точки вида $\left(r_{i}+1,1\right)$ подчинены дополнительным ограничениям, следующим из требования, чтобы все последовательные разности $r_{i+1}-r_{i}$ были нечетными целыми числами. В этом случае имеется ровно один способ дополнения ПССДРП до модифицированных, и тем самым два множества объектов находятся во взаимно однозначном соответствии.

где нижний индекс снова указывает на коэффициент при соответствующем мономе в разложении рациональной дроби в степенной ряд в точке 0. Для доказательства формулы (7) используем мультилинейность определителя и перепишем (5), (6) в виде

$$
N_{10}\left(t_{1}, t_{2}, \ldots, t_{n-1}\right)=\prod_{i=1}^{n} \oint \frac{d u_{i}}{u_{i}^{2 i-1}} \prod_{k=1}^{i-1}\left(1+t_{k} u_{i}\right) \sum_{0 \leqslant r_{0}<r_{1}<r_{2}<\cdots<r_{n-1}} \operatorname{det}_{1 \leqslant i, j \leqslant n}\left(u_{i}^{r_{j-1}}\right),
$$

где мы заменили условие $r_{0}=0$ суммированием по $r_{0} \geqslant 0$, что не изменяет результата интегрирования. Окончательно для суммы определителей получаем

$$
\sum_{0 \leqslant r_{0}<r_{1}<r_{2}<\cdots<r_{n-1}} \operatorname{det}_{1 \leqslant i, j \leqslant n}\left(u_{i}^{r_{j-1}}\right)=\prod_{1 \leqslant i<j \leqslant n} \frac{u_{j}-u_{i}}{1-u_{i} u_{j}} \prod_{i=1}^{n} \frac{1}{1-u_{i}} .
$$

Это выражение представляет собой не что иное, как сумму всех функций Шура $n$ аргументов $u_{1}, \ldots, u_{n}$, умноженных на определитель Вандермонда. Результат вычисления этой суммы является стандартным (см., например, равенство (4.17) в [4]) и приводит к равенству (7).

Для дальнейшего также представляют интерес модифицированные ПССДРП, определяемые следующим образом. Просто добавим один дополнительный шаг ко всем предыдущим ПССДРП на верхнем срезе между линиями $y=0$ и $y=1$, с весом $t_{0}$ на вертикальный шаг в этом дополнительном срезе, и наложим следующее ограничение: потребуем, чтобы новые последовательные конечные точки вида 
$\left(r_{i}+1,1\right)$ различались только на некоторые нечетные целые числа, а именно чтобы разность $r_{i+1}-r_{i}$ была нечетной при всех $i$ и выполнялось равенство $r_{1}=1$ (см. рис. 2). Причина этого состоит в том, что имеется взаимно однозначное соответствие между ними и описанными выше ПССДРП. Действительно, условие на новые конечные точки гарантирует, что ограничения этих модифицированных ПССДРП на область ниже оси $x$ будет находиться во взаимно однозначном соответствии с регулярными ПССДРП. Обозначим через $N_{10}^{\prime}\left(t_{0}, t_{1}, \ldots, t_{n-1}\right)$ соответствующий производящий многочлен.

ПримеР 2. Семь ПССДРП при $n=3$ дополняются следующим образом:

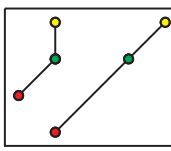

$t_{0}$

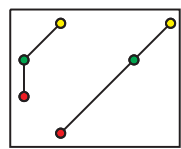

$t_{1}$

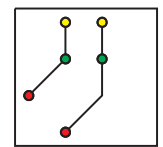

$t_{0}^{2} t_{1}$

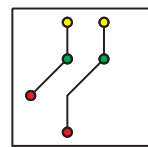

$t_{0}^{2} t_{2}$

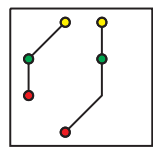

$t_{0} t_{1}^{2}$

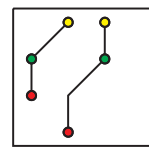

$t_{0} t_{1} t_{2}$

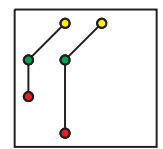

$t_{1}^{2} t_{2}$

и соответствующий производящий многочлен имеет вид

$$
N_{10}^{\prime}\left(t_{0}, t_{1}, t_{2}\right)=\left(1+t_{0} t_{1}\right)\left(t_{0}+t_{1}\right)+\left(t_{0}^{2}+t_{0} t_{1}+t_{1}^{2}\right) t_{2} .
$$

Имеем

$$
N_{10}^{\prime}\left(t_{0}, t_{1}, \ldots, t_{n-1}\right)=\left.\prod_{1 \leqslant i<j \leqslant n} \frac{\left(u_{j}-u_{i}\right)\left(1+t_{i} u_{j}\right)}{1-u_{i} u_{j}} \prod_{i=1}^{n} \frac{1+t_{0} u_{i}}{1-u_{i}^{2}}\right|_{\prod_{i=1}^{n} u_{i}^{2 i-2}} .
$$

Как и ожидалось исходя из указанного взаимно однозначного соответствия, мы воспроизвели тождество $(7)$ при $t_{0}=1$. Равенство (10) доказывается по существу тем же способом, что и $(7)$. Обозначая через $\mathcal{Q}_{i, r}$ взвешенную сумму по путям из $(i,-i)$ в $(r+1,1)$, теперь имеем

$$
\mathcal{Q}_{i, r}=\left.\prod_{k=0}^{i}\left(1+t_{k} u\right)\right|_{u^{2 i-r}}
$$

и, как и в предыдущем случае, добавляя тривиальный путь, начинающийся в начале координат и заканчивающийся в $(1,1)$ (так что дополнительная точка прибытия есть $\left.r_{0}=0\right)$, получаем

$$
N_{10}^{\prime}\left(t_{0}, t_{1}, \ldots, t_{n-1}\right)=\prod_{i=1}^{n} \oint \frac{d u_{i}}{2 \pi i u_{i}^{2 i-1}} \prod_{k=0}^{i}\left(1+t_{k} u_{i}\right) \sum_{\substack{0 \leqslant r_{0}<r_{1}<\cdots<r_{n-1} \\ r_{i+1}-r_{i} \text { нечетно }}} \operatorname{det}_{\substack{1 \leqslant i, j \leqslant n \\ \text { нетн }}}\left(u_{i}^{r_{j-1}}\right) .
$$

Заметим, что, как и раньше, мы ослабили условие $r_{0}=0$ до $r_{0} \geqslant 0$ с четным $r_{0}$ (мы ввели $r_{-1} \equiv-1$, а условие, что разность $r_{i+1}-r_{i}$ нечетна, применяется при $i=-1,0, \ldots, n-2)$. Последнюю сумму легко вычислить, используя стандартный ответ для суммы по всем функциям Шура, соответствующим четным разбиениям (см. равенство (4.29) в [4]):

$$
\sum_{\substack{0 \leqslant r_{0}<r_{1}<\cdots<r_{n-1} \\ r_{i+1}-r_{i} \text { нечетно }}} \operatorname{det}_{\substack{1 \leqslant i, j \leqslant n \\ \text { неті }}}\left(u_{i}^{r_{j-1}}\right)=\prod_{1 \leqslant i<j \leqslant n} \frac{u_{j}-u_{i}}{1-u_{i} u_{j}} \prod_{i=1}^{n} \frac{1}{1-u_{i}^{2}} .
$$


Отсюда следует равенство (10).

Для наших дальнейших целей производящий многочлен $N_{10}^{\prime}\left(t_{0}, t_{1}, \ldots, t_{n-1}\right)$ для модифицированных ПССДРП ниже будет ограничен на значения $t_{0} \equiv t$ и $t_{1}=$ $t_{2}=\cdots=t_{n-1}=\tau$. Пусть $N_{10}^{\prime}(2 n \mid t, \tau)$ обозначает соответствующий многочлен. Например, в случае $n=3$ имеем

$$
N_{10}^{\prime}(6 \mid t, \tau)=N_{10}^{\prime}(t, \tau, \tau)=t+\tau+2 t^{2} \tau+2 t \tau^{2}+\tau^{3} .
$$

Из сделанного выше замечания следует, что $N_{10}^{\prime}(2 n \mid 1, \tau)=N_{10}(2 n \mid \tau)$, поскольку модифицированные ПССДРП находятся во взаимно однозначном соответствии с ПССДРП, а значение $t=1$ соответствует случаю, когда не присваивается дополнительного веса при модификации. Поэтому при $\tau=1$ имеем $N_{10}^{\prime}(2 n \mid 1,1)=$ $N_{10}(2 n \mid 1)=A_{n}$. Это приводит, например, к равенству

$$
N_{10}^{\prime}(6 \mid 1,1)=\left.\left(t+\tau+2 t^{2} \tau+2 t \tau^{2}+\tau^{3}\right)\right|_{t=\tau=1}=7=A_{3} .
$$

Интересно, что если взять $t_{0}=0$, то единственные дающие вклад модифицированные ПССДРП - это те, у которых в последней строке имеются только диагональные шаги, что приводит к тому, что последовательные конечные точки на соответствующих ПССДРП (с удаленным верхним срезом) должны иметь нечетные целые разности, поэтому

$$
N_{10}^{\prime}\left(t_{0}=0, t_{1}, \ldots, t_{n-1}\right)=N_{10}^{\prime}\left(t_{1}, \ldots, t_{n-1}\right) .
$$

При указанном выше ограничении это дает $N_{10}^{\prime}(2 n \mid 0, \tau)=N_{10}^{\prime}(2 n-2 \mid \tau, \tau)$. В частности, если взять $\tau=1$, то получаем

$$
N_{10}^{\prime}(2 n \mid 0,1)=N_{10}^{\prime}(2 n-2 \mid 1,1)=N_{10}(2 n-2 \mid 1)=A_{n-1},
$$

например

$$
N_{10}^{\prime}(6 \mid 0,1)=\left.\left(t+\tau+2 t^{2} \tau+2 t \tau^{2}+\tau^{3}\right)\right|_{t=0, \tau=1}=2=A_{2} .
$$

Другой простой случай возникает при $t_{0} \rightarrow \infty$, когда сохраняются только конфигурации с одними вертикальными шагами в дополнительном срезе. Для этих конфигураций конечные точки $\left(r_{i}, 0\right)$ соответствующих ПССДРП опять сами по себе удовлетворяют условию четности, а именно все разности $r_{i+1}-r_{i}$ являются нечетными целыми числами:

$$
\lim _{t_{0} \rightarrow \infty} t_{0}^{-(n-1)} N_{10}^{\prime}\left(t_{0}, t_{1}, \ldots, t_{n-1}\right)=N_{10}^{\prime}\left(t_{1}, \ldots, t_{n-1}\right) .
$$

Когда все $t_{i}=1$ при $i=1,2, \ldots, n-1$, величина (15) есть не что иное, как $N_{10}^{\prime}(2 n-2 \mid 1,1)=A_{n-1}$. В случае $n=3$ это сводится к $\left.\left(t+\tau+2 t^{2} \tau+2 t \tau^{2}+\tau^{3}\right)\right|_{t^{2} ; \tau=1}=$ $2=A_{2}$.

Два частных значения $t_{0}=0$ и $t_{0}=1$ соответствуют в точности гипотезам 2 и 3 , описанным в разделе 1 . При произвольном $t$ и $\tau=1$ мы также получаем детальное перечисление ПССДРП в виде многочлена $N_{10}^{\prime}(2 n \mid t, 1)$. Коэффициенты этого 
многочлена являются, по-видимому, детальными числами МЧЗ $A_{n, k}$, которые перечисляют МЧЗ размера $n \times n$, содержащие единицу в положении $k$ верхней строки. Таким образом, имеем следующую гипотезу:

$$
N_{10}^{\prime}(2 n \mid t, 1)=\sum_{k=1}^{n} A_{n, k} t^{k-1} .
$$

Заметим, что аналогичное утверждение [14] существует для детального перечисления ПССДРП размера $2 n$ в соответствии с шагами в их верхнем срезе, а именно

$$
N_{10}(t, 1,1, \ldots, 1)=\sum_{k=1}^{n} A_{n, k} t^{k-1} .
$$

\section{3. МИНИМАЛЬНЫЕ ПОЛИНОМИАЛЬНЫЕ РЕШЕНИЯ УРАВНЕНИЯ $q \mathrm{~K} 3$}

3.1. Уравнение $q$ КЗ уровня 1 . Уравнение $q \mathrm{~K} 3$ было введено в контексте аффинных квантовых групп [9] и в связи с двумерными интегрируемыми решеточными моделями [16]. Наше основное наблюдение [11] состоит в том, что оно позволяет обобщить условие на собственный вектор основного состояния (2) на случай общих значений $q$. В настоящей работе мы изучаем уравнение $q \mathrm{~K} 3$ уровня 1 для $U_{q}\left(\widehat{\mathfrak{s l}_{2}}\right)$ (обобщения на высший ранг см. в [11]). Это уравнение основано на алгебре Темперли-Либа $T L_{2 n}(\tau)$ с генераторами $e_{i}, i=1,2, \ldots, 2 n$, подчиненными соотношениям $e_{i}^{2}=\tau e_{i}$ и $e_{i} e_{i \pm 1} e_{i}=e_{i}$ для всех $i$, с соглашением, что $e_{2 n+1} \equiv e_{1}$. Стандартное представление $T L_{2 n}(\tau)$ реализовано на типах спаривания $\pi \in \mathcal{L}_{2 n}$. Действие генератора $e_{i}$ состоит в том, что он соединяет две точки $i, i+1$ и склеивает два других конца дуги, ранее прикрепленные к ним. Если $i$ и $i+1$ уже связаны, $e_{i}$ действует как единица, умноженная на параметр $\tau$, который учитывает вес созданной таким образом петли. Этот вес на каждую петлю параметризован как

$$
\tau=-q-q^{-1}
$$

где можно интерпретировать $-q^{\epsilon}$ как вес на каждую ориентированную петлю, $\epsilon= \pm 1$ согласно ориентации, а вес $\tau$ получается суммированием по двум возможным ориентациям каждой петли.

Простое упражнение на использование генераторов алгебры Темперли-Либа состоит в построении решений уравнений унитарности и Янга-Бакстера в виде квантовой $R$-матрицы

$$
\check{R}_{i, i+1}(z, w)=\frac{q z-q^{-1} w}{q w-q^{-1} z} \mathrm{Id}+\frac{z-w}{q w-q^{-1} z} e_{i}
$$

Уравнение $q \mathrm{~K} 3$ сводится к соотношениям

$$
\begin{gathered}
\check{R}_{i, i+1}\left(z_{i+1}, z_{i}\right) \Psi\left(z_{1}, \ldots, z_{2 n}\right)=\tau_{i} \Psi\left(z_{1}, \ldots, z_{2 n}\right), \quad i=1,2, \ldots, 2 n-1 \\
\sigma \Psi\left(z_{2}, \ldots, z_{2 n}, s z_{1}\right)=c_{n} \Psi\left(z_{1}, \ldots, z_{2 n}\right)
\end{gathered}
$$


где оператор $\tau_{i}$ действует как элементарная перестановка спектральных параметров $z_{i} \leftrightarrow z_{i+1}, \sigma$ - оператор сдвига $\sigma e_{i} \sigma^{-1}=e_{i+1}$ для всех $i$, а константы $s$ и $c_{n}$ фиксированы как $s=q^{6}$ и $c_{n}=q^{3(n-1)}$.

Выражая (20) в базисе типов спаривания, получаем систему уравнений, определяющих компоненты $\Psi_{\pi}$ вектора $\Psi_{n}$, а именно:

$$
\begin{gathered}
\frac{q^{-1} z_{i+1}-q z_{i}}{z_{i+1}-z_{i}}\left(\tau_{i}-1\right) \Psi_{\pi}\left(z_{1}, \ldots, z_{2 n}\right)=\sum_{\substack{\pi^{\prime} \neq \pi \\
e_{i} \pi^{\prime}=\pi}} \Psi_{\pi^{\prime}}\left(z_{1}, \ldots, z_{2 n}\right) \\
\Psi_{\sigma(\pi)}\left(z_{2}, \ldots, z_{2 n}, q^{6} z_{1}\right)=q^{3(n-1)} \Psi_{\pi}\left(z_{1}, \ldots, z_{2 n}\right)
\end{gathered}
$$

В работах [10], [12] было показано, что пространство, обозначаемое в дальнейшем $V$, линейно порождаемое компонентами $\Psi_{\pi}$, можно просто охарактеризовать следующими условиями обращения в нуль (называемыми также условиями колеса): любой многочлен в этом пространстве обращается в нуль всякий раз, когда любая тройка циклически упорядоченных спектральных параметров принимает значения $\left(z, q^{2} z, q^{4} z\right)$. Ниже мы используем это замечание.

Приведем явный вид базисных компонент $\Psi_{\pi_{0}}$, соответствующих типу спаривания $\pi_{0}$, связывающему $i$ и $N+1-i$ :

$$
\Psi_{\pi_{0}}=\prod_{1 \leqslant i<j \leqslant n}\left(q z_{i}-q^{-1} z_{j}\right) \prod_{n+1 \leqslant i<j \leqslant N}\left(q z_{i}-q^{-1} z_{j}\right),
$$

а также полезные свойства, позволяющие получать $\Psi_{n}$ рекуррентно.

ПРЕДЛОЖЕНИЕ 1. Компоненты вектора $\Psi_{n}$, полиномиальные решения степени $n(n-1)$ уравнения qKЗ уровня 1 , нормированные условием (22), удовлетворяют рекуррентным соотношениям

$$
\left.\Psi_{\pi}\right|_{z_{i+1}=q^{2} z_{i}}=\left\{\begin{array}{lc}
0, & \pi(i) \neq i+1, \\
\prod_{j=1}^{i-1}\left(z_{i}-q^{2} z_{j}\right) \prod_{j=i+2}^{N}\left(q^{2} z_{i}-q^{-2} z_{j}\right) \times & \\
\quad \times \Psi_{\pi^{\prime}}\left(z_{1}, \ldots, z_{i-1}, z_{i+2}, \ldots, z_{N}\right), & \pi(i)=i+1,
\end{array}\right.
$$

где во втором случае $\pi^{\prime}$ получается из $\pi$ удалением дуги $(i, i+1)$.

3.2. Интегральные формулы для линейной оболочки решений. Обратимся теперь к выводу интегральных формул с множественными вычетами для векторов в пространстве, линейно порожденном компонентами $\Psi_{\pi}$ минимального полиномиального решения уравнения $q \mathrm{~K} 3$.

Пусть $z_{1}, \ldots, z_{N}$ - это $N=2 n$ комплексных переменных (спектральных параметров). Рассмотрим кратные контурные интегралы

$$
\begin{aligned}
\Psi_{a_{1}, \ldots, a_{n}}= & \prod_{1 \leqslant i<j \leqslant N}\left(q z_{i}-q^{-1} z_{j}\right) \times \\
& \times \oint \cdots \oint \prod_{\ell=1}^{n} \frac{d w_{\ell}}{2 \pi i} \frac{\prod_{1 \leqslant \ell<m \leqslant n}\left(w_{m}-w_{\ell}\right)\left(q w_{\ell}-q^{-1} w_{m}\right)}{\prod_{\ell=1}^{n} \prod_{1 \leqslant i \leqslant a_{\ell}}\left(w_{\ell}-z_{i}\right) \prod_{a_{\ell}<i \leqslant N}\left(q w_{\ell}-q^{-1} z_{i}\right)},
\end{aligned}
$$


где $\left(a_{\ell}\right)_{\ell=1, \ldots, n}-$ любая неубывающая последовательность целых чисел из множества $\{1,2, \ldots, N-1\}$. Контуры охватывают полюсы в точках $w_{i}=z_{j}$, но не охватывают полюсы в точках $w_{i}=q^{-2} z_{j}$. Эти интегралы тесно связаны с формулами для решений уравнения $q \mathrm{~K} 3$ уровня 1 в спиновом базисе (см., например, [17]). В приложении Б приводится подробное обсуждение связи между двумя типами интегралов.

Мы хотим показать следующее:

ПРЕДЛОЖЕНИЕ 2. $\Psi_{a_{1}, \ldots, a_{n}}$ есть однородный многочлен по переменным $z_{1}, \ldots, z_{N}$ степени $n(n-1)$. Более того, он удовлетворяет условиям колеса: для всех упорядоченных троек $i, j, k$

$$
\Psi_{a_{1}, \ldots, a_{n}}\left(\ldots, z_{i}=z, \ldots, z_{j}=q^{2} z, \ldots, z_{k}=q^{4} z, \ldots\right)=0, \quad 1 \leqslant i<j<k \leqslant N .
$$

Для доказательства этого утверждения сначала явно запишем формулу вычета для равенства (24):

$$
\begin{aligned}
\Psi_{a_{1}, \ldots, a_{n}}= & \prod_{1 \leqslant i<j \leqslant N}\left(q z_{i}-q^{-1} z_{j}\right) \times \\
& \times \sum_{\substack{K=\left\{k_{1}, \ldots, k_{n}\right\} \\
k_{\ell} \neq k_{m}, 1 \leqslant k_{\ell} \leqslant a_{\ell}}} \frac{\prod_{1 \leqslant \ell<m \leqslant n}\left(z_{k_{m}}-z_{k_{\ell}}\right)\left(q z_{k_{\ell}}-q^{-1} z_{k_{m}}\right)}{\prod_{\ell=1}^{n} \prod_{1 \leqslant i \leqslant a_{\ell}, i \neq k_{\ell}}\left(z_{k_{\ell}}-z_{i}\right) \prod_{a_{\ell}<i \leqslant N}\left(q z_{k_{\ell}}-q^{-1} z_{i}\right)}= \\
= & \sum_{\substack{K=\left\{k_{1}, \ldots, k_{n}\right\} \\
k_{\ell} \neq k_{m}, 1 \leqslant k_{\ell} \leqslant a_{\ell}}}(-1)^{s\left(k_{\ell}\right)} \frac{\prod_{1 \leqslant \ell<m \leqslant n}\left(q z_{k_{\ell}}-q^{-1} z_{k_{m}}\right)}{\prod_{\ell=1}^{n} \prod_{\substack{i \notin K \text { или } i>k_{\ell} \\
1 \leqslant i \leqslant a_{\ell}}}\left(z_{k_{\ell}}-z_{i}\right)} \times \\
& \quad \prod_{\substack{1 \leqslant i<j \leqslant N \\
i \notin \text { или } i=k_{\ell}, j \leqslant a_{\ell}}}\left(q z_{i}-q^{-1} z_{j}\right),
\end{aligned}
$$

где $(-1)^{s\left(k_{\ell}\right)}-$ знак перестановки, которая размещает $k_{\ell}$ в порядке возрастания.

Теперь вычислим вычет при $z_{i} \rightarrow z_{j}$. Заметим, что для того чтобы вычет слагаемого был ненулевым, по крайней мере одно из двух целых чисел $i, j$ должно принадлежать $K$. Возникают два случая.

А. Слагаемые, для которых $i$ и $j$ лежат в $K$, скажем $j=k_{\ell}$ и $i=k_{m}$ при $k_{\ell}<$ $k_{m} \leqslant a_{\ell}$ (и, как всегда, $\left.k_{m} \leqslant a_{m}\right)$. Тогда можно поменять $k_{\ell}$ и $k_{m}$ : теперь $k_{\ell}=i$, $k_{m}=j, k_{m}<k_{\ell} \leqslant a_{m}$, так что это новое слагаемое также имеет полюс при $z_{i} \rightarrow z_{j}, \mathrm{c}$ тем же вычетом, но с противоположным знаком (из-за $s\left(k_{\ell}\right)$ ), поэтому два слагаемых сокращаются.

Б. Слагаемые, для которых, скажем, только $j=k_{\ell}$ и $i \notin K$ при $i \leqslant a_{\ell}$. Рассмотрим слагаемое, где $k_{\ell}=j$ заменено на $i$ : снова кропотливый, но простой анализ позволяет проверить, что оно имеет тот же вычет с противоположным знаком.

Будучи рациональной дробью без полюсов, $\Psi_{a_{1}, \ldots, a_{n}}$ является многочленом. Свойство его однородности и значение степени получаются незамедлительно.

Рассмотрим теперь условия $1 \leqslant i<j<k \leqslant N$ и $z_{i}=z, z_{j}=q^{2} z, z_{k}=q^{4} z$ для каждого слагаемого в сумме в равенстве $(26)$ : для того чтобы множители второго типа в числителе были ненулевыми, необходимо, чтобы $i \in K$ и $j \in K$. Более того, 
чтобы множители первого типа были ненулевыми, должно быть $i=k_{m}$ и $j=k_{\ell}$ при $\ell<m$. Но тогда $j=k_{\ell} \leqslant a_{\ell} \leqslant a_{m}$ (именно здесь используется тот факт, что $\left(a_{\ell}\right)$ не убывает), так что второй множитель с индексами $i, j$ обращается в нуль. Все слагаемые обращаются в нуль, сумма является нулевой.

ПримеР 3. Чтобы $\Psi_{a_{1}, \ldots, a_{n}}$ было ненулевым, согласно формуле вычетов (26) мы должны иметь $a_{\ell} \geqslant \ell$ (в действительности $\ell \leqslant a_{\ell}<\ell+n$ ). Вычислим первую нетривиальную компоненту, т.е. $\Psi_{1,2, \ldots, n}$. Имеется единственный вычет при $w_{i}=z_{i}$, $i=1,2, \ldots, n$, и мы получаем "базисную” компоненту $\Psi_{\pi_{0}}$, даваемую формулой $(22)$.

Точное утверждение, которое может быть найдено в работе [10], состоит в том, что пространство $V$ однородных многочленов степени $n(n-1)$ по переменным $z_{1}, \ldots, z_{N}$, которые удовлетворяют условию $(25)$, имеет размерность $c_{n}=(2 n) ! /(n !(n+1) !)$ и что компоненты $\Psi_{\pi}$ решения уравнения $q \mathrm{~K} 3$ уровня 1 в базисе типов спаривания образуют базис в этом пространстве (компоненты в спиновом базисе также линейно порождают это пространство, но не являются линейно независимыми, поскольку их число есть $\left.\left(\begin{array}{c}2 n \\ n\end{array}\right)\right)$. Более того, имеется следующее предложение.

ПредлОЖеНИЕ 3. Многочлен $P \in V$ полностъю определяется своими значениями в следующих точках $c_{n}$, индексируемых типами спаривания $\pi$ :

$$
z_{i}=q^{-\epsilon_{i}(\pi)}, \quad \epsilon_{i}(\pi)=\operatorname{sign}(\pi(i)-i)= \begin{cases}+1, & \text { если } \pi \text { начинается в } i, \\ -1, & \text { если } \pi \text { оканчивается в } i .\end{cases}
$$

ДокАЗАТЕЛЬСтво. Запишем $P=\sum_{\pi} a_{\pi} \Psi_{\pi}$ и заметим, что $\Psi_{\pi^{\prime}}\left(q^{-\epsilon_{i}(\pi)}\right) \neq 0$ тогда и только тогда, когда $\pi=\pi^{\prime}$. Это легко доказать по индукции, используя (23). Действительно, выберем любую "малую дугу" из $\pi$, т.е. такое $i$, что $\pi(i)=i+$ 1. Имеем две возможности: либо $\pi^{\prime}(i) \neq i+1$, тогда $\Psi_{\pi^{\prime}}\left(q^{-\epsilon_{i}(\pi)}\right)=0$ (первый случай в равенстве $(23))$, либо $\pi(i)=i+1$, тогда можно применить второй случай в равенстве (23) и далее использовать предположение индукции для завершения доказательства. Индукция позволяет даже вычислить $C_{\pi}:=\Psi_{\pi}\left(q^{-\epsilon_{i}(\pi)}\right)$, хотя нам эти явные выражения не понадобятся:

$$
C_{\pi}=C \tau^{|\pi|}, \quad C=\left(q-q^{-1}\right)^{n(n-1)}, \quad|\pi|=n^{2}+\sum_{i=1}^{N} i \epsilon_{i}(\pi) .
$$

Возвращаясь к многочлену $P$, находим, что $a_{\pi}=P\left(q^{-\epsilon_{i}(\pi)}\right) / C_{\pi}$ и, в частности, что $P$ полностью определяется этими значениями.

Таким образом, каждый многочлен $\Psi_{a_{1}, \ldots, a_{n}}$ является линейной комбинацией компонент $\Psi_{\pi}$, а коэффициенты находятся посредством простого вычисления. В ходе доказательства мы нашли, что для вычисления $\Psi_{\pi}$ удобнее всего использовать рекуррентные соотношения, поэтому естественно попытаться поступить так же и для вычисления $\Psi_{a_{1}, \ldots, a_{n}}$. 
ПРЕДЛОЖЕНИЕ 4. Пусть $\left(a_{i}\right)$ - возрастающая последовательность. Тогда

$$
\left.\Psi_{a_{1}, \ldots, a_{n}}\right|_{z_{i+1}=q^{2} z_{i}}= \begin{cases}0, & i \notin\left\{a_{\ell}\right\}, \\ \prod_{j=1}^{i-1}\left(z_{i}-q^{2} z_{j}\right) \prod_{j=i+2}^{N}\left(q^{2} z_{i}-q^{-2} z_{j}\right) \times & \\ \times \Psi_{a_{1}, \ldots, a_{\ell-1}, a_{\ell+1}-2, \ldots, a_{n}-2}\left(z_{1}, \ldots, z_{i-1}, z_{i+2}, \ldots, z_{N}\right), & i=a_{\ell} .\end{cases}
$$

Заметим, что последовательность $\left(a_{1}, \ldots, a_{\ell-1}, a_{\ell+1}-2, \ldots, a_{n}-2\right)$ не обязательно является возрастающей (а лишь только неубывающей).

ДокАЗАТЕЛЬСтво. Предположим, что $z_{i+1}=q^{2} z_{i}$. Согласно (26), для того чтобы множители второго типа в числителе были ненулевыми, необходимо, чтобы $i \in K$, так что $i=k_{\ell}$ при некотором $\ell$ и $i+1>a_{\ell}$. Поскольку $k_{\ell} \leqslant a_{\ell}$, имеем $i=k_{\ell}=a_{\ell}$. Это доказывает первый случай в (29). Во втором случае мы видим, что если указанное $\ell$ единственно, то вклад дает только лишь вычет в $w_{\ell}=z_{i}$, так что можно вернуться к $(24)$ и проинтегрировать по $w_{\ell}$. Получаем

$$
\begin{aligned}
\Psi_{a_{1}, \ldots, a_{n}}= & \prod_{\substack{1 \leqslant j<k \leqslant N \\
(j, k) \neq(i, i+1)}}\left(q z_{j}-q^{-1} z_{k}\right) \oint \cdots \oint \prod_{\substack{1 \leqslant m \leqslant n \\
m \neq \ell}} \frac{d w_{m}}{2 \pi i} \times \\
& \times \frac{\prod_{1 \leqslant m<m^{\prime} \leqslant n}\left(w_{m^{\prime}}-w_{m}\right)\left(q w_{m}-q^{-1} w_{m^{\prime}}\right)}{\prod_{1 \leqslant m \leqslant n, m \neq \ell}^{n} \prod_{1 \leqslant j \leqslant a_{m}}\left(w_{m}-z_{j}\right) \prod_{a_{m}<j \leqslant N}\left(q w_{m}-q^{-1} z_{j}\right)} \times \\
& \times \frac{\prod_{1 \leqslant m<\ell}\left(z_{i}-w_{m}\right)\left(q w_{m}-q^{-1} z_{i}\right) \prod_{\ell<m \leqslant n}\left(w_{m}-z_{i}\right)\left(q z_{i}-q^{-1} w_{m}\right)}{\prod_{1 \leqslant j<i}\left(z_{i}-z_{j}\right) \prod_{i+1<j \leqslant N}\left(q z_{i}-q^{-1} z_{j}\right)} .
\end{aligned}
$$

Использование равенства $z_{i+1}=q^{2} z_{i}$ приводит к множественным сокращениям, и после вынесения появляющихся в $\Psi_{a_{1}, \ldots, a_{\ell-1}, a_{\ell+1}-2, \ldots, a_{n}-2}$ множителей с должной переиндексацией переменных получаем равенство (29).

Это предложение можно обобщить на произвольные последовательности (см. приложение А).

3.3. Частичные суммы в базисе типов спаривания. Рассмотрим теперь специальный класс изучаемых интегралов, соответствующих возрастающим последовательностям $\left(a_{\ell}\right)$ вида

$$
\mathcal{A}_{n}=\left\{\left(a_{\ell}\right)_{1 \leqslant \ell \leqslant n}: a_{\ell} \in\{2 \ell-2,2 \ell-1\}\right\} .
$$

Этим определяется $2^{n-1}$ различных последовательностей $\left(a_{1}=1\right.$; мы могли бы рассмотреть $a_{1}=0$, но это соответствовало бы $\left.\Psi_{0, a_{2}, \ldots, a_{n}}=0\right)$. Одно их интересное свойство состоит в том, что рекуррентное соотношение (29) выражает последовательности из $\mathcal{A}_{n}$ в терминах последовательностей из $\mathcal{A}_{n-1}$.

Рассмотрим также разбиение множества типов спаривания на подмножества, индексируемые этими же последовательностями $\left(a_{\ell}\right) \in \mathcal{A}_{n}$ :

$$
\begin{aligned}
& \mathcal{L}\left(a_{1}, \ldots, a_{n}\right)=\left\{\pi \in \mathcal{L}_{2 n}: \pi(2 m-1)>2 m-1\right. \\
& \text { тогда и только тогда, когда } \left.2 m-1 \in\left\{a_{\ell}\right\}, m=1,2, \ldots, n\right\} \text {, }
\end{aligned}
$$


т.е. множество типов спаривания, у которых нечетные вершины, являющиеся начальными точками дуг, суть в точности нечетные элементы соответствующей последовательности.

Теперь можно сформулировать следующие тождества:

$$
\Psi_{a_{1}, \ldots, a_{n}}=\sum_{\pi \in \mathcal{L}\left(a_{1}, \ldots, a_{n}\right)} \Psi_{\pi} \quad \forall\left(a_{\ell}\right) \in \mathcal{A}_{n} .
$$

Докажем эти тождества. Обе части (33) принадлежат $V$. Таким образом, достаточно показать, что они равны при значениях $c_{n}$ из $(27)$. Для этого в левой части достаточно использовать (29), т.е. использовать рекуррентные соотношения при $z_{i+1}=q^{2} z_{i}$ : действительно, для вычисления при $z_{i}=q^{-\epsilon_{i}(\pi)}$ можно выбрать малую дугу $(i, i+1)$ из $\pi$ и заметить, что $z_{i+1}=q=q^{2} q^{-1}=q^{2} z_{i}$, так что можно применить рекуррентное соотношение, которое сводится к тому же вычислению (с типом спаривания, полученным из $\pi$ удалением малой дуги $(i, i+1))$ в размеpe $n-1$. Таким образом, нам надо установить те же рекуррентные соотношения для правой части (33). Мы используем равенство (23). Положим $z_{i+1}=q^{2} z_{i}$, и пусть сначала $i \notin\left\{a_{\ell}\right\}$. Если $i$ нечетно, это означает, что все $i$ не являются начальной точкой ни для одного $\pi \in \mathcal{L}\left(a_{\ell}\right)$. Если $i$ четно, то $i+1 \in\left\{a_{\ell}\right\}$ (ср. (31)) и, таким образом, все $\pi \in \mathcal{L}\left(a_{\ell}\right)$ имеют начальной точкой $i+1$. В обоих случаях заключаем, что у них нет малой дуги, соединяющей $i$ и $i+1$, а потому они равны нулю, так что правая часть равенства (33) равна нулю. Пусть теперь $i=a_{\ell}$. Все $\pi \in \mathcal{L}\left(a_{1}, \ldots, a_{n}\right)$ распадаются на две категории: те, для которых $\pi(i) \neq i+1$ и которые поэтому не дают вклада в сумму при $z_{i+1}=q^{2} z_{i}$, и те, для которых $\pi(i)=i+1$. Легко проверить, что последние находятся во взаимно однозначном соответствии с $\pi^{\prime} \in \mathcal{L}\left(a_{1}, \ldots, a_{\ell-1}, a_{\ell+1}-2, \ldots, a_{n}-2\right)$, причем взаимно однозначное соответствие устанавливается, как обычно, посредством удаления малой дуги $(i, i+1)$. Поскольку множители в (23) и (29) одинаковы, заключаем, что рекуррентные соотношения, которым удовлетворяют обе части (29), совпадают. Начальные условия при $n=0$ также совпадают: $\Psi_{\varnothing}=1$.

ПримеР 4. При $n=3$ имеем $\mathcal{A}_{3}=\{(1,3,5),(1,3,4),(1,2,5),(1,2,4)\}$ и разбиение типов спаривания имеет вид

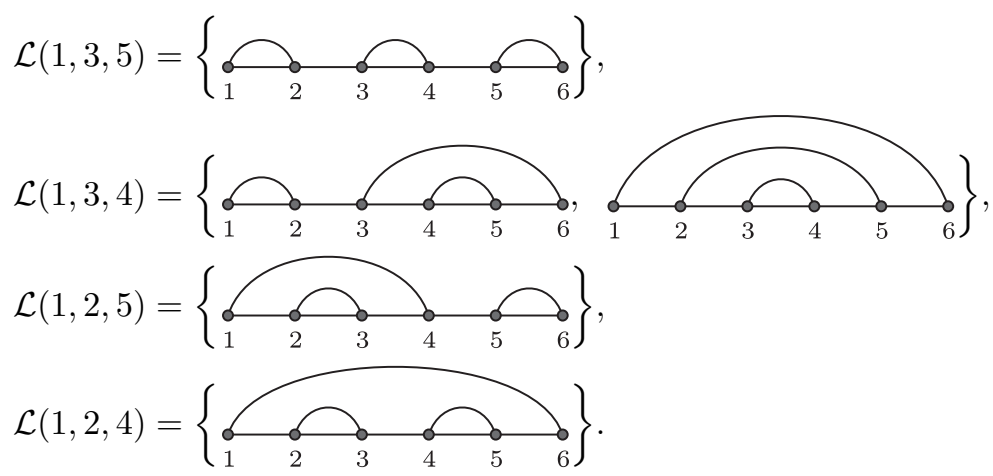

Заметим, что $\mathcal{L}(1,3, \ldots, 2 n-1)$ всегда является синглетом, $\pi(2 \ell-1)=2 \ell, \ell=$ $1,2, \ldots, n$, и что $\mathcal{L}(1,2, \ldots, 2 n-2)$ всегда является синглетом $\pi(1)=N, \pi(2 \ell)=2 \ell+1$, 
$\ell=1,2, \ldots, n-1$. Вообще, $\mathcal{L}\left(a_{1}, \ldots, a_{n}\right)$ содержит типы спаривания, начальными точками которых являются в точности числа $a_{\ell}$, но могут содержать и другие элементы.

3.4. Однородный предел. Рассмотрим, наконец, однородный предел $z_{1}=\cdots$ $\cdots=z_{2 n}=1$. Перепишем равенство (24) для этого случая и нормируем его так, что $\Psi_{\pi_{0}}=\left(q-q^{-1}\right)^{n(n-1)}$ :

$$
\frac{\Psi_{a_{1}, \ldots, a_{n}}}{\Psi_{\pi_{0}}}=\left(q-q^{-1}\right)^{n^{2}} \oint \cdots \oint \prod_{\ell=1}^{n} \frac{d w_{\ell}}{2 \pi i} \frac{\prod_{1 \leqslant \ell<m \leqslant n}\left(w_{m}-w_{\ell}\right)\left(q w_{\ell}-q^{-1} w_{m}\right)}{\prod_{\ell=1}^{n}\left(w_{\ell}-1\right)^{a_{\ell}}\left(q w_{\ell}-q^{-1}\right)^{2 n-a_{\ell}}}
$$

Замена переменных $w_{\ell}=\left(1-q^{-1} u_{\ell}\right) /\left(1-q u_{\ell}\right)$ дает

$$
\frac{\Psi_{a_{1}, \ldots, a_{n}}}{\Psi_{\pi_{0}}}=\oint \cdots \oint \prod_{\ell=1}^{n} \frac{d u_{\ell}}{2 \pi i u_{\ell}^{a_{\ell}}} \prod_{1 \leqslant \ell<m \leqslant n}\left(u_{m}-u_{\ell}\right)\left(1+u_{\ell} u_{m}+\tau u_{m}\right),
$$

где контуры охватывают точку 0 , а $\tau=-q-q^{-1}$.

Как следствие, сумма всех компонент равна

$$
\sum_{\pi \in \mathcal{L}_{2 n}} \frac{\Psi_{\pi}}{\Psi_{\pi_{0}}}=\oint \cdots \oint \prod_{\ell=1}^{n} \frac{\left(1+u_{\ell}\right) d u_{\ell}}{2 \pi i u_{\ell}^{2 \ell-1}} \prod_{1 \leqslant \ell<m \leqslant n}\left(u_{m}-u_{\ell}\right)\left(1+u_{\ell} u_{m}+\tau u_{m}\right) .
$$

Это соотношение получается суммированием равенства (35) по всем последовательностям $\left(a_{\ell}\right) \in \mathcal{A}_{n}$ и применением равенства $(33)$.

ПримеР 5. При $n=3$ имеем $\Psi_{1,3,5}=\tau^{3}+\tau, \Psi_{1,3,4}=\tau^{2}+1, \Psi_{1,2,5}=\tau^{2}, \Psi_{1,2,4}=$ $2 \tau$, и полная сумма имеет вид $\sum_{\pi} \Psi_{\pi}=\tau^{3}+2 \tau^{2}+3 \tau+1$.

\section{4. ОТ ИНТЕГРАЛЬНЫХ ФОРМУЛ НАЗАД К ПССДРП}

4.1. Детальная $q$ КЗ-ПССДРП гипотеза. Рассмотрим снова производящий ряд $N_{10}^{\prime}$ для модифицированных ПССДРП для значений $t_{0}=t$ и $t_{1}=t_{2}=\cdots=$ $t_{n-1}=\tau$ : согласно равенству $(10)$ он имеет вид

$$
N_{10}^{\prime}(2 n \mid t, \tau)=\oint \cdots \oint \prod_{\ell=1}^{n} \frac{d u_{\ell}\left(1+t u_{\ell}\right)\left(1+\tau u_{\ell}\right)^{\ell-1}}{2 \pi i u_{\ell}^{2 \ell-1}} \frac{\prod_{1 \leqslant \ell<m \leqslant n}\left(u_{m}-u_{\ell}\right)}{\prod_{1 \leqslant \ell \leqslant m \leqslant n}\left(1-u_{\ell} u_{m}\right)} .
$$

С другой стороны, с точки зрения частичных сумм, входящих в (33), представляется естественным определить следующий производящий ряд:

$$
\widehat{N}_{10}^{\prime}(2 n \mid t, \tau):=\sum_{\left(a_{\ell}\right) \in A} t^{\sum_{\ell}\left(2 \ell-1-a_{\ell}\right)} \sum_{\pi \in \mathcal{L}\left(a_{1}, \ldots, a_{n}\right)} \frac{\Psi_{\pi}}{\Psi_{\pi_{0}}} .
$$

С помощью равенства (35) получаем формулу

$$
\widehat{N}_{10}^{\prime}(2 n \mid t, \tau)=\oint \cdots \oint \prod_{\ell=1}^{n} \frac{d u_{\ell}\left(1+t u_{\ell}\right)}{2 \pi i u_{\ell}^{2 \ell-1}} \prod_{1 \leqslant \ell<m \leqslant n}\left(u_{m}-u_{\ell}\right)\left(1+\tau u_{m}+u_{\ell} u_{m}\right) .
$$


Заметим, что при $t=1$ многочлен $\widehat{N}_{10}^{\prime}(2 n \mid 1, \tau)$ является просто суммой всех компонент в петлевом базисе. Таким образом, содержание гипотезы ПССДРП из работы [1] заключается в том, что $\widehat{N}_{10}^{\prime}(2 n \mid 1, \tau)=N_{10}^{\prime}(2 n \mid 1, \tau)$.

Заметим еще, что при $t=0$ многочлен $\widehat{N}_{10}^{\prime}(2 n \mid 0, \tau)$ является наибольшей компонентой $\Psi_{\max } / \Psi_{\pi_{0}}$, тогда как $N_{10}^{\prime}(2 n \mid 0, \tau)=N_{10}^{\prime}(2 n-2 \mid \tau, \tau)$. В частности, при $\tau=1$ обе они равны $A_{n-1}$.

На основе этих частных случаев и численных экспериментов мы пришли к гипотезе, что $N_{10}^{\prime}(2 n \mid t, \tau)=\widehat{N}_{10}^{\prime}(2 n \mid t, \tau)$ для всех значений параметров. Например, при $n=3$ имеем

$$
\widehat{N}_{10}^{\prime}(6 \mid t, \tau)=\Psi_{1,3,5}+t\left(\Psi_{1,3,4}+\Psi_{1,2,5}\right)+t^{2} \Psi_{1,2,4}=\tau^{3}+2 t \tau^{2}+2 t^{2} \tau+\tau+t,
$$

что совпадает с $N_{10}^{\prime}(6 \mid t, \tau)$ из раздела 2 .

4.2. Попытки доказательства гипотезы и предполагаемое тождество. Чтобы доказать эту гипотезу, достаточно доказать совпадение равенств (37) и (39). С учетом свободно-фермионной природы ПССДРП естественно антисимметризовать эти выражения. Получаем следующие формулы:

$$
\begin{aligned}
& \left\{\prod_{1 \leqslant \ell \leqslant m \leqslant n}\left(1-u_{\ell} u_{m}\right) \operatorname{AS}\left(\prod_{i=1}^{n} u_{\ell}^{-2 \ell+2} \prod_{1 \leqslant \ell<m \leqslant n}\left(1+u_{\ell} u_{m}+\tau u_{m}\right)\right)\right\}_{\leqslant 0}= \\
& =\operatorname{AS}\left(\prod_{\ell=1}^{n}\left(u_{\ell}^{-1}\left(\tau+u_{\ell}^{-1}\right)\right)^{\ell-1}\right)=\prod_{1 \leqslant \ell<m \leqslant n}\left(u_{m}^{-1}-u_{\ell}^{-1}\right)\left(\tau+u_{\ell}^{-1}+u_{m}^{-1}\right),
\end{aligned}
$$

где AS обозначает антисимметризацию по отношению к перестановкам переменных $u$, а именно:

$$
\operatorname{AS}\left(f\left(u_{1}, \ldots, u_{n}\right)\right)=\sum_{\sigma \in \mathcal{S}_{n}}(-1)^{\sigma} f\left(u_{\sigma(1)}, \ldots, u_{\sigma(n)}\right),
$$

а нижний индекс " $\leqslant 0$ " указывает, что надо удерживать только члены с отрицательными или нулевыми степенями по всем переменным $u_{\ell}$. Последнее равенство в (40) элементарно, но антисимметризация в первом равенстве нетривиальна. Легко проверить, что из (40) следует, что симметризованные подынтегральные выражения в (37) и (39) совпадают, так что эти интегралы равны ${ }^{2}$.

Эквивалентным образом можно переписать (40) в виде интегрального тождества

$$
\begin{aligned}
\oint \cdots \oint & \prod_{\ell=1}^{n} \frac{d u_{\ell}}{2 \pi i} \frac{1-x u_{\ell}^{2}}{2 \pi i u_{\ell}^{2 \ell} \times} \\
& \times \operatorname{det}_{1 \leqslant \ell, m \leqslant n}\left(\frac{1}{1-\alpha_{\ell} u_{m}}\right) \prod_{1 \leqslant \ell<m \leqslant n}\left(1+\tau u_{m}+x u_{\ell} u_{m}\right)\left(1-x u_{\ell} u_{m}\right)= \\
= & \prod_{\ell=1}^{n} \alpha_{\ell} \prod_{1 \leqslant \ell<m \leqslant n}\left(\alpha_{m}-\alpha_{\ell}\right)\left(\tau+\alpha_{\ell}+\alpha_{m}\right) .
\end{aligned}
$$

${ }^{2)}$ После завершения данной статьи Д. Цайльбергер получил доказательство равенства (40), которое можно найти на его сайте http://www.math.rutgers.edu/ zeilberg/pj.html. 
По поводу этого тождества можно сделать два существенных замечания. Определитель в подынтегральном выражении, с точностью до определителя Вандермонда для переменных $u$, является производящей функцией для многочленов Шура от этих переменных, которые сами по себе линейно порождают пространство симметричных многочленов от этих переменных. Более того, результат является очевидным образом не зависящим от параметра $x$. Комбинируя два этих факта, мы видим, что если заменить определитель любым симметричным многочленом переменных $u$, умноженным на определитель Вандермонда для тех же $u$, то интеграл остается не зависящим от $x$. При подходящем выборе симметричной функции, приравнивая значения при $x=1$ и $x=0$, мы получаем совпадение (37) и (39).

4.3. Перспективы. Высокая степень подобия равенств (37) и (39) весьма красноречива. Однако эти формулы также напоминают формулы из работы [18], а это означает, что их доказательство может оказаться сложнее, чем это кажется, поэтому мы решили отложить его. В заключение приведем несколько общих замечаний.

Прежде всего заметим, что основной результат данной работы - гипотеза, подробно изложенная в п. $4.1,-$ связывает объекты четырех разных типов: для параметра $\tau$ в общем положении она связывает $\tau$-взвешенное исчисление ПССДРП с дополнительным весом $t$, зависящим от четности их конечных точек (или, что эквивалентно, дополненных ПССДРП со специальным весом для последней строки), и сумму компонент полиномиального решения уравнения $q \mathrm{~K} 3$ уровня 1 (при $\tau=-q-q^{-1}$ ) в базисе типов спаривания, где дополнительный вес $t$ зависит от четности конечных точек в петлях. При ограничении на значение $\tau=1$ она также связывает указанные объекты с $t$-детализированным перечислением МЧЗ и с суммой компонент основного состояния в петлевой модели Темперли-Либа, в которой все спектральные параметры, кроме одного, взяты равными единице, причем последний играет роль $t$.

Ни одна из этих связей полностью не понята. Введение спектральных параметров - основная идея работы [6] - пролило некоторый свет на этот предмет, но не ясно, как внедрить полный набор параметров в гипотезу ПССДРП. Первая очевидная мысль, которая могла бы прийти на ум, состоит в том, чтобы использовать интегрируемости модели замощения ромбами и снабдить ее спектральными параметрами, но наши попытки оказались безуспешными. Заметим, однако, что такая связь между статистической суммой неоднородной модели замощений ромбами шестиугольника размера $a \times b \times c$ и фактическими компонентами $\Psi$, отвечающими типам спаривания с тремя наборами вложенных дуг, была установлена в работе [19].

Методы данной работы можно обобщить и на другие граничные условия. Например, в работе [2] была высказана гипотеза, что сумма компонент решения уравнения $q \mathrm{~K} 3$ уровня 1 с открытыми (отражающими) границами равна в однородном пределе и для произвольного $q$ производящему многочлену для $\tau$-взвешенных (возможно, модифицированных) циклически-симметричных транспонированно дополнительных разбиений плоскости, а именно циклически и отражательно-инвариантных замощений шестиугольника ромбами (возможно, с треугольной дырой в центре). Более общие граничные условия рассматривались в работе [20], и мы 
полагаем, что интегральные формулы, аналогичные полученным в данной работе, должны существовать для различных правил сумм для компонент полиномиального решения $\Psi$ соответствующего уравнения $q \mathrm{~K} 3$, и, возможно, они допускают связь с $\tau$-перечислением разбиений плоскости с подходящими симметриями. Можно также поразмышлять о том, как провести обобщение на случаи высших рангов и высших спинов, рассматривавшиеся в работах [11] и [21]. Заметим, однако, что в случаях высших рангов или спинов найденные обобщенные правила сумм Разумова-Строгонова приводят к последовательностям целых чисел, для которых пока не существует комбинаторной интерпретации, и остается открытой проблема нахождения тех обобщений МЧЗ/ПУП или ПССДРП, которым они могут соответствовать.

Наконец, отметим высокую степень схожести данной работы с недавней статьей [22]. Это будет предметом отдельного обсуждения [23].

ПРИЛОЖКЕНИЕ А

\section{Вычисление коэффициентов $\Psi_{a_{1}, \ldots, a_{n}}$ в базисе типов спаривания}

Равенство (29), дающее рекуррентные соотношения для $\Psi_{a_{1}, \ldots, a_{n}}$ в случае возрастающей последовательности $\left(a_{\ell}\right)$, можно обобщить на произвольные неубывающие последовательности:

$$
\begin{aligned}
\left.\Psi_{a_{1}, \ldots,} \underbrace{i, \ldots, i, \ldots, a_{n}}_{k}\right|_{z_{i+1}=q^{2} z_{i}}=\prod_{j=1}^{i-1}\left(z_{i}-q^{2} z_{j}\right) \prod_{j=i+2}^{N}\left(q^{2} z_{i}-q^{-2} z_{j}\right) \times \\
\quad \times \frac{q^{k}-q^{-k}}{q-q^{-1}} \Psi_{a_{1}, \ldots, \underbrace{i-1, \ldots, i-1}_{k-1}, \ldots, a_{n}-2}\left(z_{1}, \ldots, z_{i-1}, z_{i+2}, \ldots, z_{N}\right) .
\end{aligned}
$$

Доказательство проводится в том же духе, что и для (29).

Как следствие, если мы вычисляем коэффициенты компонент $\Psi_{\pi}$ в базисе типов спаривания в точках (27), использование равенства (42) дает следующее: прежде всего, ненулевые коэффициенты соответствуют типам спаривания, у которых малые дуги начинаются в точках из $\left\{a_{\ell}\right\}$; для каждого из них использование рекурсии подразумевает удаление (скажем, самой левой) малой дуги на каждом шаге; число $k$, соответствующее началу, определяет коэффициент $U_{k-1}=\left(q^{k}-q^{-k}\right) /\left(q-q^{-1}\right)$, и сумма чисел, соответствующих началу и концу, уменьшенная на единицу, добавляется к вершине слева.

Этот процесс удобно описать графически следующим образом. Сдвинем переменные $a_{i}$ на полшага вправо и нанесем маленький маркер (кружок), указывающий на число букв $a_{\ell}$ в этом месте. Рекурсионный процесс состоит в удалении одной малой дуги: если внутри нет маркера, то вклад равен нулю; в противном случае вычтем единицу из значения $k$ маркера внутри, быть может удаляя его, если он обращается в нуль, и умножим вклад на соответствующий коэффициент $U_{k-1}$. 
Пример 6. Для $\Psi_{1,3,4,5}$ ненулевые коэффициенты имеют вид

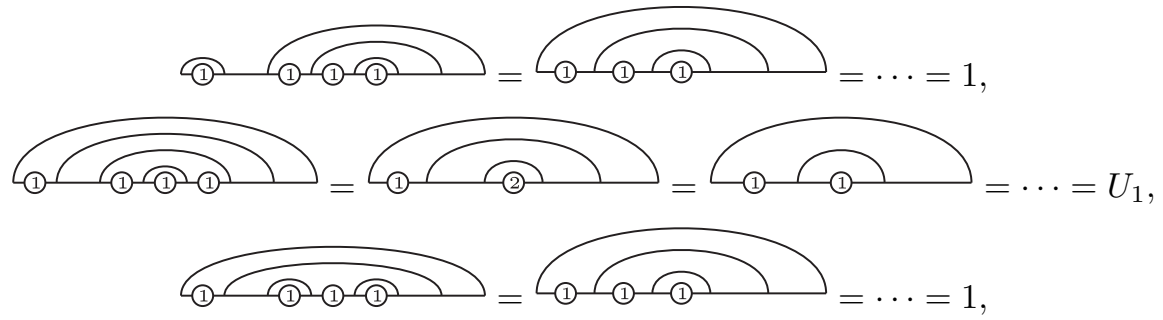

а для $\Psi_{2,4,5,5}-$
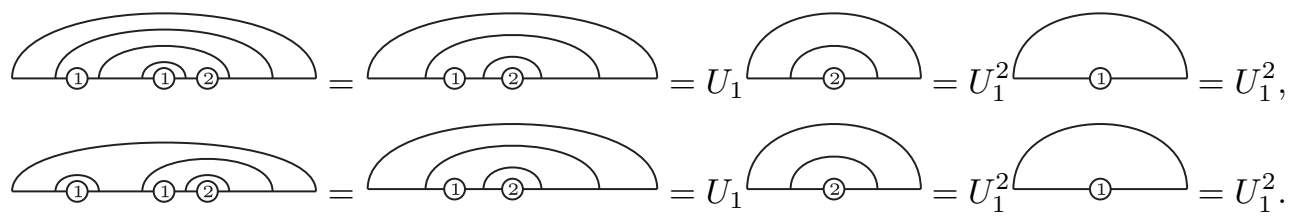

Рекурсию можно, конечно, разрешить, и окончательная формула для коэффициентов при компонентах $\Psi_{\pi}$ в $\Psi_{a_{1}, \ldots, a_{n}}$ имеет вид

$$
\prod_{i<\pi(i)} U_{\#\left\{\ell: i \leqslant a_{\ell}<\pi(i)\right\}-(\pi(i)-i+1) / 2},
$$

где $U_{-1}=0, U_{0}=1, U_{1}=-\tau, U_{2}=\tau^{2}-1$ и т.д.

В качестве проверки заметим, что отсюда можно воспроизвести равенство (33). Действительно, если $a_{\ell} \in\{2 \ell-2,2 \ell-1\}$, то

$$
\#\left\{\ell: i \leqslant a_{\ell}<\pi(i)\right\}= \begin{cases}\frac{\pi(i)-i+1}{2}, & i \text { четное и } \pi(i)-1 \in\left\{a_{\ell}\right\} \\ \frac{\pi(i)-i-1}{2} & \text { или } i \text { нечетное и } i \in\left\{a_{\ell}\right\},\end{cases}
$$

\section{Компоненты в спиновом базисе}

ПРИЛОЖКЕНИЕ Б

Приведенное в [17] выражение для решения уравнения $q \mathrm{~K} 3$ уровня 1 в спиновом базисе (базисе последовательностей из $n$ плюсов и $n$ минусов), после избавления от различных множителей, несущественных для наших целей, имеет вид

$$
\begin{aligned}
& \widetilde{\Psi}_{a_{1}, \ldots, a_{n}}=\left(q-q^{-1}\right)^{n} \prod_{1 \leqslant i<j \leqslant N}\left(q z_{i}-q^{-1} z_{j}\right) \times \\
& \quad \times \oint \cdots \oint \prod_{\ell=1}^{n} \frac{w_{\ell} d w_{\ell}}{2 \pi i} \frac{\prod_{1 \leqslant \ell<m \leqslant n}\left(w_{m}-w_{\ell}\right)\left(q w_{\ell}-q^{-1} w_{m}\right)}{\prod_{\ell=1}^{n} \prod_{1 \leqslant i \leqslant a_{\ell}}\left(w_{\ell}-z_{i}\right) \prod_{a_{\ell} \leqslant i \leqslant N}\left(q w_{\ell}-q^{-1} z_{i}\right)},
\end{aligned}
$$

где $a_{\ell}-$ положения плюсов. Замечая, что $\left(q-q^{-1}\right) w_{\ell}=\left(q w_{\ell}-q^{-1} z_{i}\right)-q^{-1}\left(w_{\ell}-z_{i}\right)$, и сравнивая с (24), находим

$$
\widetilde{\Psi}_{a_{1}, \ldots, a_{n}}=\sum_{\varepsilon_{1}, \ldots, \varepsilon_{n} \in\{0,1\}}(-q)^{-\sum_{i} \varepsilon_{i}} \Psi_{a_{1}-\varepsilon_{1}, \ldots, a_{n}-\varepsilon_{n}} .
$$


В частности, в однородном пределе получаем интегральную формулу, аналогичную (35):

$$
\widetilde{\Psi}_{a_{1}, \ldots, a_{n}}=\oint \ldots \oint \prod_{\ell=1}^{n} \frac{d u_{\ell}\left(1-q^{-1} u_{\ell}\right)}{2 \pi i u_{\ell}^{a_{\ell}}} \prod_{1 \leqslant \ell<m \leqslant n}\left(u_{m}-u_{\ell}\right)\left(1+u_{\ell} u_{m}+\tau u_{m}\right) .
$$

Заметим, что формула для $a_{\ell}=2 \ell-1$ ("наибольшей компоненты" в спиновом базисе $(+-\cdots+-))$ является специальным случаем $t=-q^{-1}$ для "детализированного перечисления" (39).

Не лишена интереса возможность воспроизвести смену базиса от типов спаривания к спинам в нашем формализме. Действительно, используя метод приложения $\mathrm{A}$, можно ограничиться рассмотрением вклада малой дуги $(i, i+1)$. Смысл равенства (43) состоит в том, что каждый плюс можно сдвинуть или на полшага вправо с весом 1 , или на полшага влево с весом $-q^{-1}$. В результате получим четыре локальные конфигурации вблизи $i, i+1$ :

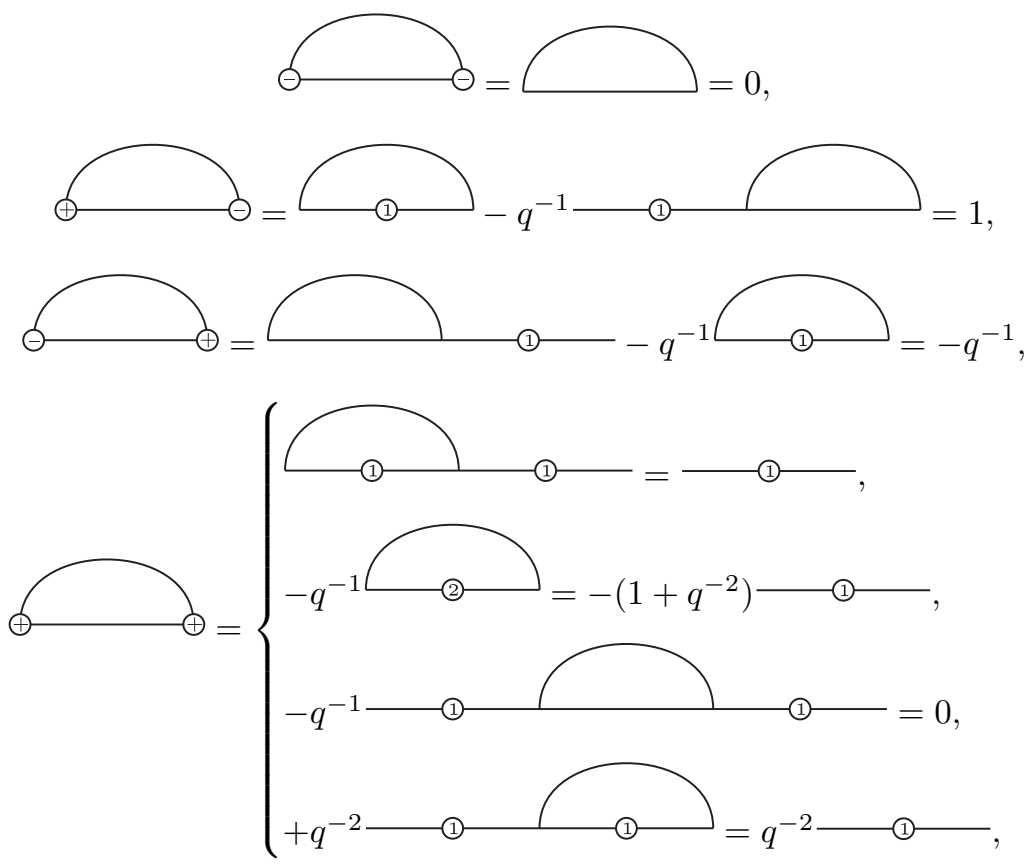

и обратим внимание на то, что в последнем случае все вклады сокращаются, так что мы воспроизводим обычное правило, состоящее в том, что дуга приобретает вес 1 , если ее начало соответствует плюсу, а ее конец - минусу, вес $-q^{-1}$, если ее начало соответствует минусу, а конец - плюсу, и вес 0 в остальных случаях.

Благодарности. Авторы были поддержаны со стороны European Marie Curie Research Training Networks "ENIGMA" MRT-CT-2004-5652, "ENRAGE" MRTN-CT2004-005616, ESF программы "MISGAM" и ANR программы "GIMP" ANR-05-BLAN0029-01. П. Зинн-Жюстен признателен организаторам семинара "Классические и квантовые интегрируемые системы", где эта работа была закончена. 


\section{Список литературы}

[1] P. Di Francesco, J. Stat. Mech., 2006, № 9, P09008; arXiv : cond-mat/0607499.

[2] P. Di Francesco, J. Stat. Mech., 2007, № 1, P01024; arXiv: math-ph/0611012.

[3] M. T. Batchelor, J. de Gier, B. Nienhuis, J. Phys. A, 34 (2001), L265-L270; arXiv: cond-mat/0101385.

[4] D. Bressoud, Proofs and Confirmations: The Story of the Alternating Sign Matrix Conjecture, Cambridge Univ. Press., Cambridge, 1999.

[5] А. В. Разумов, Ю. Г. Строганов, ТМФ, 138 (2004), 395-400; arXiv: math/0104216.

[6] P. Di Francesco, P. Zinn-Justin, Electron. J. Comb., 12:1 (2005), R6; arXiv: math-ph/0410061.

[7] А. Г. Изергин, ДАН СССР, 297:2 (1987), 331-333; V. Korepin, Comm. Math. Phys., 86 (1982), 391-418.

[8] G. Kuperberg, Ann. Math. (2), 156:3 (2002), 835-866; arXiv: math/0008184.

[9] I. B. Frenkel, N. Yu. Reshetikhin, Comm. Math. Phys., 146:1 (1992), 1-60.

[10] V. Pasquier, Ann. Henri Poincaré, 7:3 (2006), 397-421; arXiv: cond-mat/0506075.

[11] P. Di Francesco, P. Zinn-Justin, J. Phys. A, 38:48 (2005), L815-L822; arXiv: math-ph/0508059.

[12] M. Kasatani, V. Pasquier, Comm. Math. Phys., 276:2 (2007), 397-435; arXiv: cond-mat/0608160.

[13] P. Di Francesco, J. Phys. A, 38 (2005), 6091-6120; arXiv: math-ph/0504032v2; J. Stat. Mech., 2005, № 11, P11003; arXiv: math-ph/0509011v3.

[14] D. Robbins, Math. Intelligencer, 13:2 (1991), 12-19.

[15] B. Lindström, Bull. London Math. Soc., 5 (1973), 85-90; I. Gessel, G. Viennot, Adv. Math., $\mathbf{5 8 : 3}$ (1985), 300-321.

[16] F. A. Smirnov, J. Phys. A, 19:10 (1986), L575-L578.

[17] M. Jimbo, T. Miwa, Algebraic Analysis of Solvable Lattice Models, CBMS Regional Conference Series in Mathematics, 85, AMS, Providence, RI, 1995.

[18] D. Zeilberger, Electron. J. Combin., 3:2 (1996), R13; arXiv: math/9407211.

[19] P. Zinn-Justin, Electron. J. Combin., 13:1 (2006), R110; arXiv: math/0607183.

[20] P. Di Francesco, P. Zinn-Justin, From orbital varieties to alternating sign matrices, arXiv: math-ph/0512047.

[21] P. Zinn-Justin, Combinatorial point for higher spin loop models, arXiv: math-ph/0603018.

[22] A. V. Razumov, Yu. G. Stroganov, J. Stat. Mech., 2006, №7, P07004; arXiv: math-ph/0605004.

[23] A. V. Razumov, Yu. G. Stroganov, P. Zinn-Justin, J. Phys. A, 40:39 (2007), 11827-11847; arXiv: $0704.3542 \mathrm{v} 3$. 